REFLEXIONES 

Fecha de entrega: 27 de noviembre de 2009

Fecha de aprobación: 8 de marzo de 2010

\title{
DE LAS CONDICIONES DE \\ POSIBILIDAD PARA VIVIR JUNTOS EN COLOMBIA: ENTRE TRAMAS DE SIGNIFICACIONES QUE SOSTIENEN EL CONFLICTO COLOMBIANO Y LA CONSTRUCCIÓN SIMBÓLICA DE UNA ESFERA PÚBLICA
}

\author{
"ABOUT THE CONDITIONS FOR A POSSIBILITY OF LIVING \\ TOGETHER (SHARING THE SAME SPACE) IN COLOMBIA: \\ BETWEEN MEANING PLOTS (INTRIGUES) SUPPORTING \\ (FEEDING) THE COLOMBIAN CONFLICT AND \\ A PUBLIC SPHERE SYMBOLICAL BUILDING"
}

José Gabriel Cristancho Altuzarra*

\section{Resumen}

En este trabajo se aborda el siguiente interrogante: ¿cuáles son las condiciones de posibilidad para vivir juntos en Colombia? El autor de este trabajo señala el carácter filosófico de este problema: se trata de un punto de intersección entre dos campos de investigación de la filosofía: la ética y la política; pero además de esto, aplicado a un caso concreto —Colombia—, con el propósito de aportar, desde la filosofía, una reflexión seria sobre problemáticas sociales que vive el país.

Para resolver este interrogante, se parte de la hipótesis de que los conflictos entre los seres humanos dependen, entre otras cosas, de tramas simbólicas que circulan entre los sujetos que lo sustentan; así, de esta manera, el trabajo explicitará algunas tramas simbólicas que

Licenciado en Filosofía, magíster en Filosofía Latinoamericana, doctorando en Educación. correo electrjogacral@gmail.com 
"le dan suelo" el conflicto armado colombiano y el rol de la memoria en construcción de esa trama. En seguida, se señala la importancia de la construcción de una trama simbólica que posibilitaría la sana convivencia entre los miembros de una sociedad (una esfera pública) y las circunstancias azarosas que han dificultado que esa esfera se componga como trama simbólica.

\title{
Palabras clave
}

Ética, política, conflicto armado, memorias de la violencia política

\begin{abstract}
The author of this work pose the question: what are the possibility conditions for to live together in Colombia? So, it shows the philosophic condition of that problem: it is a point of intersection between two areas of investigation of the philosophy: ethics and politic; but, besides, this question is applied to a concrete case -Colombia-, with the purpose of the philosophy contribute to study the social problems of our country.

For to solve this question, the author begins with the hypothesis of conflicts between the human beings depend, of symbolic networks that circulate between the subjects; so, the work explains some symbolic networks that found the Colombian warlike conflict and the role of the memory in the construction of those networks. Then, is shows the importance of the construction of a symbolic network that will make possible the safe life together between the members of a society (a public sphere) and the hazardous circumstances that have interfered that that sphere make up as symbolic network.
\end{abstract}

\section{Key words}

Ethics, politic, warlike conflict, memories of the politic violence.

\section{Introducción}

La ética y la política son dos campos de estudio que desde la antiguedad han preocupado a los filósofos. Hay una pregunta elemental y fundamental que surge de la intersección de estos dos campos; esa inquietud es: ¿cuáles son las condiciones de posibilidad para vivir juntos? Implícita o explícitamente este interrogante se ha planteado de diversas formas a lo largo de la historia del pensamiento occidental y en diversos lugares. Se acentúa su formulación en la medida en que existe el conflicto y la violencia entre las naciones, lo cual hace de esta pregunta una cuestión de carácter cosmopolita. Pero también, se inflama su razón de ser cuando lo que está en cuestión es la convivencia al interior de un grupo humano o, en términos actuales, al interior de un Estado.

Esa es la situación que vive el Estado colombiano desde hace décadas: existe un conflicto interno, en el cual distintos grupos defienden sus intereses desde la lucha armada ilegal (guerrillas, paramilitares, mafias del narcotráfico). En el medio, la población civil padece sufrimientos que van desde desplazamientos forzados hasta los peores atentados que vulneran su dignidad. También existen 
desproporciones sociales que no parecen resolverse: Colombia es uno de los países con mayores índices de desigualdad en el mundo. Esta situación, entre otras, hace totalmente vigente que se reflexione por las condiciones de posibilidad para vivir juntos en Colombia.

Este problema, en general, daría para hacer una investigación de grandes proporciones, no sólo por las situaciones tan complejas que padece nuestro Estado, sino porque parece una cuestión difícil de resolverse ${ }^{1}$. Así, no se trata de una cuestión simple y pretender resolverla en un ensayo de pocas páginas sería atrevido. Sin embargo, algunos autores ${ }^{2}$ que se han dado a la tarea de reflexionar la cuestión del conflicto en Colombia nos hacen pensar que si el conflicto interno - condición que imposibilita la convivencia entre colombianos-, está sustentado en tramas simbólicas que lo movilizan (Blair, s.f.), una de las condiciones de posibilidad de vivir juntos tiene que ver con otra construcción simbólica: la de una esfera pública (Pecaut, 2001); estas construcciones de sentido se dan en el proceso de la constitución de la subjetividad de los individuos y en el cual la memoria es elemento fundamental.

Pues bien, el objetivo de este ensayo es desarrollar y explicar esta hipótesis, como uno de los aportes a esa gran pregunta por las

1 En efecto, aunque dicen los entendidos en antropología que el ser humano, el homo sapiens sapiens lleva sobre el planeta miles de años, los distintos conflictos y perversidades en contra de los semejantes que se dieron en las guerras mundiales del siglo pasado y en procesos de descolonización en África, así como conflictos entre Israel y Palestina, y la guerra en la antigua Yugoslavia, por citar algunos ejemplos, hacen pensar que, en el aspecto de la vida en común, el ser humano no ha logrado evolucionar.

2 Me refiero a Elsa Blair, Martha Cabrera, Adolfo Chaparro y Daniel Pécaut. Algunos de los textos que inspiraron este ensayo serán citados a lo largo de este trabajo y pueden verse en la bibliografía. condiciones de posibilidad para vivir juntos en Colombia. Para desarrollar esta hipótesis, en primer lugar, siguiendo las sugerencias de Elsa Blair y algunas insinuaciones de Chaparro y Daniel Pécaut, procuraré explicitar algunas tramas simbólicas que sustentan el conflicto armado y el rol de la memoria en esa construcción; en seguida, siguiendo a Pécaut, señalaré la importancia de la construcción de la esfera pública y las circunstancias azarosas que han dificultado que esa esfera se componga.

\section{Algunos rasgos de la dimensión simbólica del conflicto interno colombiano}

Algunos entendidos han insistido en que el conflicto interno colombiano es difícil de caracterizar. Pécaut, por ejemplo, insiste en que "la combinación de dimensiones heterogéneas oscurecen la aprehensión del conflicto al hacer menos visible su eje central" (Pécaut, 2004, p. 90). Antropólogos, historiadores y politólogos se han dado a la tarea de aportar a la comprensión del asunto, pero la obscuridad del fenómeno sigue persistiendo.

Sin embargo, el ser humano es, como diría Aristóteles, el viviente simbólico por naturaleza. Esto significa que toda experiencia vivida es asimilada cuando se le da un sentido, esto es, cuando se le integra en la existencia a través de significados. Esta integración hace parte de la construcción de la subjetividad: las construcciones simbólicas construyen nuestro mundo interior, nuestra subjetividad.

Ahora bien, la memoria es una de las dimensiones fundamentales de la construcción de la subjetividad; Blair, entiende la memoria "como un recurso de interpretación y reinterpretación de la realidad donde están en constante negociación las diferentes versiones del pasado (...) la memoria acude al relato para dar una posición, una historia, y una identidad 
al sujeto, pero necesariamente en relación con los otros" (Blair, 2002, p. 24).

En efecto, la memoria es resultado de la intención discursiva del sujeto por ubicarse en el mundo, integrándose en una línea de sentido que completa las vivencias desde el pasado, a través del presente y dirigiéndose hacia el futuro. Esta línea de sentido es fruto, no sólo de las vivencias personales, sino de los aprendizajes culturales que incluyen relatos de vivencias de otros, relatos religiosos, históricos o míticos, marcos teóricos, científicos o filosóficos, que conforman la "memoria colectiva".

Entonces, la memoria es depositaria de tramas simbólicas que configuran la subjetividad y orientan nuestras acciones; en este sentido, comprender el conflicto armado implica comprender que hay una construcción de subjetividades que se ha sustentado en una trama simbólica que es necesario desvelar. De ahí que aunque Pécaut señale dificultades para comprender el conflicto, Blair propone que se recupere una línea de trabajo: analizar el conflicto desde su dimensión cultural; en efecto, según ella, "el análisis actual precisa esclarecer, además de los contextos materiales de la violencia (tradicionalmente analizados) los contextos donde su dimensión simbólica tiene un sentido, es decir, los contextos culturales a partir de los cuales la violencia es ejecutada, leída, pensada, narrada y significada" (Blair, s.f. n. 9, p. 18).

Esta referencia reafirma las reflexiones que se vienen sosteniendo: que la violencia o cualquier fenómeno social es consecuencia, entre otras, de una serie de significaciones que es preciso desvelar: "el análisis cultural exige mirar el campo de las representaciones mentales que acompañan los actos de violencia, es decir, su dimensión simbólica: sentidos, representaciones, imaginarios, significaciones, tramas discursivas de los fenómenos violentos" (Blair, s.f. p. 15).
Si se entienden bien estas reflexiones, esto significa que hay una trama de significaciones que le da sustento al conflicto armado; pero esta trama, sin duda alguna, hace parte de la construcción de la subjetividad de los individuos. Pues bien; tratemos de desentrañar esa dimensión simbólica que ha configurado memorias y subjetividades y que le ha dado un "sentido" y sostiene el conflicto armado colombiano; en este ensayo me referiré fundamentalmente a una trama de sentido en particular: la construcción simbólica del amigo-enemigo. Veamos:

Un conflicto armado, sea el lugar que sea, o la época que sea, sólo puede sustentarse cuando existe un enfrentamiento de intereses de dos o más bandos. En esta situación tiene que darse la construcción simbólica del otro como "enemigo": ¿cuál es la trama de símbolos y de construcciones imaginarias que sustentan la eliminación del otro, que sustentan la construcción del otro como un enemigo en nuestro contexto de conflicto? Señalemos algunos ejemplos.

Por el lado de las guerrillas de izquierda existe la dualidad opresor/oprimido, construcción simbólica que hace representar la lucha armada como lucha revolucionaria. En efecto, uno de los ideólogos e inspiradores de las guerrillas latinoamericanas es el Che Guevara; en uno de sus escritos puede verse esa construcción simbólica. En efecto, dice que el guerrillero "es el combatiente de la libertad por excelencia, es el elegido del pueblo" (Guevara, 1959a, párrafo 1), un reformador de la sociedad que lucha contra una clase social que oprime: "el guerrillero es un reformador social que empuña sus armas como protesta airada del pueblo contra sus opresores" (Guevara, 1959a, párrafo 9).

Por el lado de la institucionalidad se han construido los opuestos democracia/terrorismo, dualidad que representa la lucha armada como lucha contra el terrorismo, como lucha 
por la "seguridad democrática". Esto hace parte de una construcción simbólica institucional liderada por el gobierno desde el 2002: una re-construcción simbólica del conflicto a partir del imaginario global del terrorismo:

El gobierno Uribe no sólo desecha las tesis de las causas objetivas del conflicto y las teorías redistributivas sobre su solución, sino que cambia radicalmente el lenguaje histórico y redefine el conflicto como lucha contra el terrorismo, facilitando las medidas económicas y legislativas que conlleva esta decisión en cuanto a la definición soberana del enemigo interno (Chaparro, 2005, p. 456).

Los medios masivos de información y entretenimiento han servido de mediación para que esta construcción simbólica se mantenga. Un ejemplo de esto es el slogan de la mayor parte de propagandas televisivas, radiales y de algunas vallas presentes en las vías del país que legitiman el accionar armado del ejército nacional: "Los héroes en Colombia sí existen". "Viaje tranquilo; su ejército está en la vía”.

Estos discursos y construcciones simbólicas han ocasionado que el conflicto se mantenga en la medida en que se ha construido el símbolo amigo-enemigo. Daniel Pécaut escribió en 1999: "He mencionado la eventualidad de que guerrilla y paramilitares quieran suscitar una polarización de la sociedad (...). La polarización supone que la población se convenza de la existencia de una división amigo-enemigo que sobredetermine todos los aspectos de la vida social. Por suerte estamos lejos de esto" (Pecaut, 1999, p. 34). Pasados casi 11 años ya no podemos decir esto. El proceso de desmovilización de las AUC iniciado en el 2002, la polémica ley de justicia y paz, los escándalos del ingreso del paramilitarismo en el Congreso (cuando los máximos jefes paramilitares ingresaron al edificio del Congreso y relación de congresistas con paramilitares), la política de seguridad democrática de los últimos ocho años y el estatus de terroristas sólo para los grupos insurgentes de izquierda lograron crear la trama simbólica de una polarización que si bien no cataloga como "amigos" a los paramilitares, sí describe como "enemigos" bien concretos y únicos a los grupos guerrilleros, sobre todo a las FARC.

Sobre este punto es fundamental tener en cuenta el reciente artículo de opinión de María Jimena Dussan publicado por la revista Semana sobre la cantidad de críticas en contra de los Moncayo después de la liberación de este secuestrado que estuvo en cautiverio durante 12 años:

el propio sargento Moncayo, se debe estar preguntando cuál pudo haber sido su gran pecado para merecer tanta ignominia, tanto desprecio, tanto odio visceral y tanta intolerancia. La respuesta, mi querido soldado, es solo una: mientras usted estuvo secuestrado en la selva y pasó por los horrores más inhumanos, este país no sólo cambió tecnológicamente; su mayor cambio fue en su comportamiento cultural, a pesar de que se registraron importantes avances en el tema de la seguridad y en la lucha contra las Farc. En estos doce años nuestro imaginario cultural se ha ido construyendo con base en percepciones que se han vuelto verdades, así no lo sean. Esos postulados, derivados en su gran mayoría de la seguridad democrática, nos han vuelto una sociedad más uniforme y por lo tanto menos tolerante; una sociedad más segura pero políticamente primaria -el único discurso permitido es el que habla de acabar 
al enemigo, las Farc, y a todo el que esté con él-; y una sociedad aparentemente más exitosa pero más corrupta (Dussan, 2010, s.p.).

Estos elementos son suficientes para señalar que en Colombia hay construcciones simbólicas que no cohesionan a las personas. Por el contrario, las dividen. Estas tramas de sentido sustentan el conflicto, lo alimentan y parecen perpetuarlo. ¿Cómo pueden ser estas construcciones simbólicas condición de posibilidad para vivir juntos? Esta pregunta, en el fondo, nos interroga a la vez por cuáles son las condiciones de posibilidad de una construcción simbólica que facilitaría la convivencia entre los seres humanos. Esto nos ocupará a continuación ${ }^{3}$.

\section{Los avatares de una construcción de la esfera pública como dimensión simbólica que posibilitaría el vivir juntos}

En el anterior apartado hemos evidenciado la importancia de la construcción de la subjetividad, el rol de la memoria y de la dimensión simbólica del ser humano en el desarrollo del conflicto colombiano como condición que imposibilita el vivir juntos. Nos orientamos ahora a las condiciones que lo facilitarían. Esta cuestión, al igual que la dimensión simbólica humana, es una cuestión natural: el hombre es un viviente comunitario por

3 Un tema que podría entrar en esta reflexión y que dejo abierto para el desarrollo de otra investigación es, cómo en estos últimos años ha procurado construirse una simbología de enemistad entre lo colombiano y lo venezolano a raíz de distintos factores políticos que se han visto en los medios masivos de información y entretenimiento. Esta trama de sentido también puede imposibilitar una convivencia entre las dos naciones. naturaleza, como dijo también Aristóteles ${ }^{4}$. Así como parece imposible que el hombre viva con otros, más imposible aún es que viva totalmente aislado, sin otros.

Ahora bien, ¿existe relación entre subjetividad y la cuestión del vivir juntos? Sin duda. La construcción de la subjetividad implica la construcción de una dimensión privada, la interioridad, o, si se quiere, la intimidad; pero también la construcción de la subjetividad se da en relación con otros; implica la composición de una dimensión pública.

Ambas dimensiones están cruzadas por sentidos, por significados. Si el hombre es un animal comunitario, si está en continua relación con otros, esto nos remite a uno de esos significados: la noción moderna de lo público. Dentro de ella hay otros sentidos o representaciones; Pécaut insiste que la noción de lo público tiene que ver con la aceptación de un principio de semejanza entre todos los miembros, la existencia de debates en un espacio no organizado por el Estado, la existencia real de una sociedad civil, entendida como una sociedad relativamente autónoma de unas estructuras institucionales políticas y el reconocimiento de un mundo común en donde "vivimos juntos" (Pécaut, 2001, p. 3).

La cuestión de vivir juntos tiene que ver, entonces, con una trama simbólica: por un lado el comprenderse semejante a otros; por otro, comprenderse en un mundo compartido, en un mundo común que cohesiona la unidad. Ese mundo compartido es una construcción simbólica subjetiva pero también intersubjetiva: como dice Pécaut, "lo público está circunscrito a una historicidad concreta en donde se elaboran formas de interacción habituales, así como represen-

$4 \quad$ Đ ¥nqrwpoj fÚsei politikÕn zùon $\left[{ }^{\mathrm{TM}_{\mathrm{Sti}}}\right.$ ] (Aristóteles, Política I, i, 1253a 3. Introducción, versión y notas de Antonio Gómez Robledo. México: UNAM, 2000, p. 4). 
taciones simbólicas de la unidad nacional" (Pécaut, 2001, p. 5). Algunos entendidos, entre ellos Pécaut, hablan de esfera pública; en este ensayo consideraremos que la esfera pública es una construcción simbólica, una trama que da sentido y que abarca ese mundo compartido y ese comprenderse semejante que establece una conexión social y moral entre las personas.

Sin duda que una construcción simbólica como ésta, como la esfera pública, puede ayudar a los seres humanos a vivir juntos. La pregunta, entonces, es: ¿cuáles han sido los avatares de esta construcción simbólica de la esfera pública en Colombia? Veamos:

El anterior apartado ya nos ha mostrado cómo el mismo conflicto interno y la trama de significado que lo sustenta ha dificultado la construcción simbólica de la esfera pública; sin embargo, ha habido otros factores que se han interpuesto en este proceso. Pécaut señala que:

La construcción de un "interés público" hace parte también de las cargas del Estado. Cuando éste no logra imponer dicho interés más allá de las reivindicaciones y exigencias de cada grupo, o cuando deja que sea la lógica del mercado la que asigna recursos y bienes colectivos su legitimidad se desmorona. A la vez que esto sucede, a la sociedad civil se le dificultará cada vez más salvaguardar el sentido de lo público, puesto que cada grupo particular querrá hacer valer su interés o su "identidad" por encima de cualquier proyecto colectivo (Pécaut, 2001, p. 6).

La anterior reflexión nos muestra dos cosas fundamentales: primero, la carga que tiene el Estado con relación a la creación de esa esfera pública; el Estado, como institución, no ha sabido - o no ha tenido la voluntad (?) de sortear este papel; pueda ser que se haya "legitimado" usando la mediatización de la "seguridad democrática", como mencionamos anteriormente; pero desde hace décadas ha permitido que la lógica del mercado asigne los recursos, con la continua política de privatización y el abuso del recurso público ${ }^{5}$. Existen muchos grupos particulares que hacen valer su interés por encima de un proyecto de nación. De hecho, no puede decirse que exista un proyecto nacional. Por eso Pécaut señala que "la sociedad civil organizada sólo tiene en común su desconfianza hacia el Estado" (Pécaut, 2001, p. 15).

Esto se reafirma, aún más, en la siguiente reflexión:

El repliegue sobre los intereses privados, las dificultades que encuentra la acción colectiva, la difusión

$5 \quad$ Los índices de corrupción política son muy elevados. Es curioso que sean prácticamente nulas las referencias a este tema en los textos que se han trabajado (Pécaut, Cabrera, Blair, etc.). Aunque se hable de memorias de la violencia, suele hacerse un énfasis casi exclusivo en la memoria de los conflictos como tales y en sus secuelas, mas no en la memoria de los factores que la ocasionan. Una memoria obligada, sin duda, sería, las memorias sobre la corrupción en Colombia: ¿qué tanto se recuerda que hay corrupción en Colombia y que esa corrupción es un factor fundamental en los problemas sociales, incluida la violencia? Sin embargo, podría pensarse también que no es que la memoria sobre la corrupción haya dejado de existir; quizá también se pueda hablar de una trivialización de la corrupción política, en la medida en que la generalidad de la población ha ido aceptando e integrando costumbres como la compraventa de votos, la aceptación de que la función de los políticos se reduzca a buscar empleo a los "amigos" y el otorgamiento de contratos a instituciones que tienen convenio político o económico con los dirigentes. Estas prácticas han consolidado una construcción simbólica sobre la corrupción que la ha legitimado. 
de formas de incivismo no son el resultado de un defecto de "socialización". Son los efectos de crisis institucionales y representan maneras de adaptación racional a situaciones donde no se da la cooperación ni la certeza sobre la estabilidad de los acuerdos (Pécaut, 2001, p. 11).

Finalmente, no puede ignorarse que el modo de vida occidental contemporáneo ha minado las posibilidades de una forma de vida comunitaria. En efecto, así como la trama simbólica de la violencia se manifiesta y se compone a través de prácticas por parte de los actores, la construcción simbólica de una esfera pública no surge solamente por la recepción pasiva de representaciones sociales (medios de comunicación o dispositivos educativos); toda construcción simbólica se alimenta de hábitos y formas rituales que dan sentido y que son sustentadas por la significación. En este sentido, nos recuerda Pécaut:

Varios autores - entre ellos Habermas-, habían mostrado la primacía de la "razón instrumental", cuya consecuencia era la "colonización del mundo vivido". Esta tendencia no ha hecho más que incrementarse. Ya no es posible hablar del espacio público sin mencionar el papel de los medios de comunicación, los cuales están ante todo regidos por la lógica del mercado (Pécaut, 2001, p. 7).

La primacía de la razón instrumental se refiere, sin duda, al avance de la sociedad globalizada y globalizante que se ha impuesto en el desarrollo tardío del capitalismo, sistema que ha fundamentado su lógica en construcciones simbólicas individualistas. En el afán de integrarse al grupo de los países desarrollados, Colombia, como otros países, ha asimilado las políticas del sistema económico imperante, sin cuestionar siquiera las posibles causas de desintegración de los individuos. Hasta cierto punto esta desintegración también es necesaria y fundamental para que las leyes del mercado se mantengan (Abbott, J. \& Achbar, M., 2003) ${ }^{6}$.

En estas circunstancias, ese comprenderse semejante a otros que señalamos al principio puede darse, pero entendido como un aglomerado de individuos cuyas relaciones logran con dificultad traspasar las relaciones comerciales. Por otra parte, la representación simbólica de un mundo común también se resquebraja por los intereses individuales o grupales sobre la propiedad de los medios de producción o del mismo mundo.

En este sentido y, retomando la problemática de la violencia, quizás la capacidad de reconocer y sentir el dolor de los otros depende, en gran medida, de la construcción de un mundo común. El que no siente el dolor del otro no tiene interés alguno en manifestarse en contra de él; a su vez si no ha sido posible una construcción de un mundo común, imposible es sentir el dolor del otro. Esto está emparentado por la imposibilidad de que el Estado (entendido como el sistema de instituciones políticas) haya sido capaz de cohesionar un proyecto de nación y sí haya provocado la pérdida de confianza en él por la corrupción; pero también, de la "sociedad civil".

Todo esto, sumado a la contracción social provocada por la violencia que no ha terminado, ha hecho azarosa la construcción de la esfera de lo público como dimensión simbólica que haría posible la convivencia entre los colombianos.

6 Sobre este punto son interesantes las reflexiones que se hacen en el documental The Corporation de J. Abbott y M. Achbar. 


\section{Perspectivas}

En la reflexión que se ha ejercido en este ensayo se ha mostrado el rol fundamental de las representaciones simbólicas en el desarrollo del conflicto armado colombiano; a su vez, se evidencia el papel que estas construcciones de sentido tienen en la memoria y en la construcción de la subjetividad. Sin embargo, todo esto se ha planteado en el marco de la inquietud fruto de la intersección de la ética y la política: la pregunta por las condiciones de posibilidad de vivir juntos $y$, de manera particular, en Colombia.

El análisis ha mostrado también los avatares de una trama de significación que cohesione a los colombianos, una construcción simbólica de la esfera de lo público. Las dificultades y obstáculos a los que se ve expuesto este proceso muestran un horizonte difícil, pero también nos muestra la opción que tenemos los intelectuales, a través de la educación, de mostrar esas posibilidades, por un lado, para deconstruir las tramas de significado que imposibilitan la convivencia entre nosotros y entre los demás países.

Este ensayo ha sido un abrebocas a este campo de investigación: siguiendo las indicaciones de Blair, la de retomar el análisis de la violencia desde la dimensión cultural, se trata de desentrañar las tramas de sentido que sustentan el conflicto colombiano; sus construcciones simbólicas pueden encontrarse en muchos aspectos; hemos sugerido, por ejemplo, abordar las tramas de significado que circulan en los medios masivos de información y entretenimiento; este es un campo de investigación amplio; en el momento el autor se está orientando, en esta línea, hacia la investigación del discurso cinematográfico.

Por otro, el campo de investigación que sigue abierto es el que interroga por las posibilidades de pensar otras construcciones de sentido capaces de transgredir todos aquellos obstáculos que impiden el vivir juntos. Podríamos preguntarnos, por ejemplo, cómo se ha asimilado en el ámbito privado la problemática social colombiana y si se ha asimilado al punto de que impulse y configure una esfera pública; Pécaut se pregunta: "cómo lograr que surja un espacio público orientado hacia este bien (la paz como "bien público")" (Pécaut, 2001, p. 16).

Se trata, en síntesis, de reivindicar la tarea del pensar a la que Heidegger nos abocó al ver los peligros del predominio de la ciencia y de la técnica. Al fin y al cabo, el pensador puede avizorar desde lo alto un horizonte que haga posible que el ser humano trascienda sus límites, pues el pensar mismo es condición de posibilidad para que el ser humano se trascienda a sí mismo.

\section{Referencias}

Abbott, J. \& Achbar, M. (2003). The Corporation. (135 min.). Canadá; USA.

Blair, E. (2002, junio-diciembre). Memoria y Narrativa: La puesta del dolor en la escena pública. En Estudios Políticos, $2 l$, 9-28. Medellín.

Blair, E. (s.f.). La violencia frente a los nuevos lugares y/o los otros de la cultura. Falta ciudad, editorial

Cabrera, M. (2005-2006). Exceso y defecto de la memoria: violencia política, terror, visibilidad e invisibilidad. En Revista Oasis, 11. 39-55.

Chaparro, A. (2005, agosto). Procesos de subjetivación, conflicto armado y construcción del Estado-nación en Colombia. En Revista Socio - Jurídicos. Vol. 7 Número Especial: Justicia transicional: memoria colectiva, reparación, justicia y democracia. Bogotá: Editorial Universidad del Rosario. 
Dussan, M.J. (2010). Todos contra Moncayo. En Semana. No. 137091. Abril 2 de 2010. Recuperado el 5 de abril de 2010 en http:// www.semana.com/noticias-opinion/ todos-contra-moncayo/137091.aspx

Guevara, E. (1959a). ¿Qué es un guerrillero? Recuperado el 15 de marzo de 2009 en http://www.marxists.org/espanol/ guevara/59-quees.htm

Pécaut, D. (2001). Crisis y construcción de lo público. En V Encuentro Iberoameri- cano del Tercer Sector. Lo público: Una pregunta desde la sociedad civil, Bogotá.

Pécaut, D. (1999). Las configuraciones del espacio, el tiempo y la subjetividad en un contexto de terror: el caso colombiano. En Revista de Antropología, 35, 8-35.

Pécaut, D. (2004). Memoria imposible, historia imposible, olvido imposible. En Memorias en conflicto, Aspectos de la violencia política contemporánea. Lima: IEP-IFEA. 